\title{
Knockdown of 60S Ribosomal Protein L14-2 \\ Reveals Their Potential Regulatory Roles in Enhancing Drought and Salt Tolerance in Cotton
}

Margaret shiraku ( $\nabla$ smargy10@yahoo.com )

Chinese Academy of Agricultural Sciences https://orcid.org/0000-0002-2878-5933

Richard Odongo Magwanga

Chinese Academy of Agricultural Sciences

Xiaoyan Cai

Chinese Academy of Agricultural Sciences

Joy Nyangasi Kirungu

Chinese Academy of Agricultural Sciences

Yanchao Xu

Chinese Academy of Agricultural Sciences

Teame Gereziher Mehari

Chinese Academy of Agricultural Sciences

Yuqing Hou

Chinese Academy of Agricultural Sciences

Yuhong Wang

Chinese Academy of Agricultural Sciences

Renhai Peng

Chinese Academy of Agricultural Sciences

Kunbo Wang

Chinese Academy of Agricultural Sciences

Zhongli Zhou

Chinese Academy of Agricultural Sciences

Liu Fang

Chinese Academy of Agricultural Sciences

\section{Research}

Keywords: Cotton, Transcription factor, Ribosomal protein Large, Abiotic stress, Virus-induced gene silencing

Posted Date: March 15th, 2021 
DOI: https://doi.org/10.21203/rs.3.rs-291618/v1

License: (c) (1) This work is licensed under a Creative Commons Attribution 4.0 International License. Read Full License 


\section{Abstract}

\section{Background}

Cotton is an important economic crop and the primary source of natural fiber. The effects of drought and salt stresses threaten strong fiber and large quantity production. However, due to the ever-changing climatic conditions, plants have evolved various mechanisms to cope with the effects of various stress factors. One of the plant's transcription factors with positive effects in alleviating effects of drought and salt stresses is the Ribosomal protein Large $(R P L)$ gene families. This has prompted the functional characterization of the RPL14B gene previously identified in the QTL region as a candidate gene that responds to stress and initiates mechanisms that enhance stress tolerance.

Results

Comprehensive identification and functional analysis were conducted in this study, in which 26,8 , and 5 proteins containing the RPL14B domain were identified in G. hirsutum, G. raimondii, and G. arboreum, respectively. Moreover, Cis-regulatory elements associated with the RPL genes were identified. The Myb binding sites (MBS), Myb, Abscisic acid-responsive element (ABRE), CAAT-box, TATA box, TGACG-motif, and CGTCA-motif responsive to Meja, and TCA- motif responsive to salicylic acid were identified. Validation of the candidate gene through virus-induced gene silencing (VIGS) revealed that the Gh_D01G0234 (RPL14B) knockdown significantly affected the cotton seedling's performance under drought/ salt stress conditions as evidenced by a significant reduction in various morphological and physiological traits. Moreover, antioxidant enzyme levels were significantly reduced in VIGS-plants, with substantially higher oxidant enzyme levels, as evidenced by the higher concentration level of Malondialdehyde (MDA).

Conclusion

The results revealed the potential role of the gene, and it can be further exploited to breed climate-smart cotton varieties resilient to drought and salt stress conditions

\section{Highlights}

- Cotton is the source of natural fiber. However, due to climate change, drought and salt stresses threaten strong fiber and large quantity production.

- RPL 14B gene was previously identified in the QTL map as a candidate gene for drought stress tolerance.

- Virus-Induced Gene Silencing (VIGS) revealed that the Gh_D01G0234 (RPL14B) knockdown, significantly affected the performance of the cotton seedlings under drought and salt stress conditions.

\section{Background}


Cotton is an essential plant worldwide (Campbell et al., 2010), and primarily, it is the natural source of fiber (Haigler, Betancur, Stiff, \& Tuttle, 2012), oil (Singh, Kendall, Hake, \& Ramkumar, 2013), and protein for animals feeds (Rogers, Poore, \& Paschal, 2002). However, high quantity and quality cotton production are ever declining due to the effects of abiotic stress factors like cold, drought, and salinity (Magwanga, Lu, Kirungu, Diouf, et al., 2018). The adverse effect of drought and salinity stress conditions has intensified with ever-changing climate conditions. Therefore, improvement of drought and salinity stress tolerance could reduce osmotic stress-induced yield loss. Previous studies have demonstrated that drought and salt stresses induce the expression of osmotic stress-associated genes. Ribosomal protein large (RPL) is a gene family that was previously thought to be primarily involved in enhancing homeostasis inside the ribosomal complex and protein biosynthesis (Chaillou, 2020). However, recent studies have demonstrated that abiotic stress factors regulate the transcription of genes coding for the RPL protein (Horiguchi, Van Lijsebettens, Candela, Micol, \& Tsukaya, 2012). For example, GmRPL37 is highly expressed during cold stress in soybean and positively regulated cold tolerance (K. Kim et al., 2004). Overexpression of RPL44 in Aspergillus glaucus enhanced drought and salt stress tolerance (Liu et al., 2014). Overexpression of RPL23A in transgenic rice increased the rice plant's water use efficiency and improved tolerance to drought stress (Moin, Bakshi, Madhav, \& Kirti, 2017). In 2008, Rogalski found out that RPL33 in tobacco plants was not essential when plants are growing in suitable growing conditions but were crucial in enhancing acclimation to cold stress (Rogalski, Schöttler, Thiele, Schulze, \& Bock, 2008). The RPL genes are characterized by multiple abiotic stress and phytohormones cis-elements in their transcription regions, which respond specifically to stress and signal molecules (Moin et al., 2017, 2016; Saha et al., 2017). MBS (Myb binding site) and Low-Temperature Response (LTR), among others, are widely distributed in the putative promoter region of RPL genes. These responsive elements are associated with genes responsive to drought and cold stress, respectively (Zou et al., 2011). Their presence in the promoter region of the RPL supports the fact that these genes are involved in abiotic stress response and tolerance.

QTL mapping is one of the strategies developed and currently used to identify genes involved in different plant pathways (C.-K. Kim et al., 2019). In previous research done by Magwanga et al., a $\mathrm{BC}_{2} \mathrm{~F}_{2}$ population generated from $G$. tomentosum as the donor parent, well-known to have a high tolerance to drought and G. hirsutum, the recurrent parent, which is widely cultivated because of high yielding but susceptible to drought and salt stress, was developed. A high-density genetic linkage map was developed by adopting genotyping by sequencing (GBS), integrating genotype and phenotype (Magwanga, Lu, Kirungu, Diouf, et al., 2018). Several stable Quantitative trait loci (QTLs) were identified and grouped into three main clusters focusing on the physiological related QTLs contributed by the donor parent $G$. tomentosum which were cell membrane stability (CMS), chlorophyll content, and saturated leaf weight (SLW). Within the QTL regions, 89 Genes were mined, among them Gh_D01G0234 (RPL14B). Further, they analyzed the genes through RNA sequence data from the public domain database and validation through qRT-PCR under drought and found the gene to be upregulated (MAGWANGA et al., 2020). 
The mined genes were of interest because they were contributed by the donor parent $G$. tomentosum. $G$. tomentosum is wild cotton species whose natural habitat is saline and dry, thus tolerant to drought and salt stress conditions (Oluoch et al., 2016). Wild plant species are known to have traits that, when introgressed into the cultivated cultivars, can improve the plant tolerance to abiotic stress conditions and increase yield quantity and quality (Des Roches et al., 2018; Wang et al., 2021). Therefore, in this research work, characterization and functional validation of the 60S Ribosomal Protein L14-2 (RPL 14B) gene in cotton is important. In addition, the various bioinformatics analysis about Cis-regulatory elements, GO terms, KEGG, conserved motif, gene structure, and phylogenetic relationship were performed. Moreover, the expression profiles of the RPL $14 B$ gene family were carried on the various tissues under drought and salt stresses. Gh_D01G0234 gene was further validated through virus-induced gene silencing (VIGS), and the VIG-plants were evaluated under drought and salt stress conditions. The results revealed that the $R P L 14 B$ gene could have potential and significant roles in stress tolerance. This work provides

fundamental steps for future exploration of the $R P L 14 B$ genes in improving cotton germplasm to develop climate-smart cotton varieties resilient to drought and salt stress factors.

\section{Materials And Methods}

\section{Phylogenetic tree analysis and physio-chemical properties of RPL14B protein}

The sequences of the RPL14B were obtained from the three cotton genomes, A, D and AD. The tetraploid (AD) cotton genome was G. hirsutum, G. barbadense, G. tomentosum, G. mustelinum, and G. darwinii, while the diploid cotton (A and D) was G. arboreum and G. raimondii. The tetraploid cotton protein sequences were obtained from their respective genome databases through the Blastp program, while the diploid protein sequences were downloaded from the cotton functional genomics database (https://cottonfgd.org/CottonFGD). Using the Pfam Scan (https://www.ebi.ac.uk/Tools/pfa/pfamscan/) and SMART search (http://smart.emblheidelberg. de/smart/) we queried all the genes in order to identify the RLP14B genes with Pfam domain PF01929. ClustalX and MEGA 7 programs were used to conduct multiple sequence alignments of the RPL14B protein sequences and construct the phylogenetic tree (Kumar, Stecher, \& Tamura, 2016; Thompson, Gibson, \& Higgins, 2003). The physical and chemical aspects of the $R P L 14 B$ gene family were determined using the CottonFGD database.

\section{Gene structure, motif identification and Gene Ontology enrichment analysis}

Online tools determined the gene structure and conserved motif of RPL14B genes, the gene structure displayer server (http://gsds.cbi.pku.edu.cn), and MEME Suite (http://meme-suite.org/) (Bailey et al., 2009; Hu et al., 2015). We employed GO Analysis Toolkit and Database, AgriGO v2.0, to conduct gene ontology annotation of the RPL14B genes (www.bioinfo.cau.edu.cn/agriGO) (Tian et al., 2017).

\section{Chromosomal allocation, cis-regulatory element prediction, and subcellular localization prediction}

The information on RPL14B chromosome position was downloaded from the CottonGen website and using the chromosome information, mapping of the genes was done by TbTools (Chen et al., 2020). The 
subcellular localization of the RLP14B proteins was determined through Wolfpsort (https://www.wolfpsort.hgc.jp/) (Hortona, Park, Obayashi, \& Nakai, 2006). G.hirsutum, G.raimondii, and G.arboreum 2000 bp nucleotide sequence, retrieved from the cotton FGD database, was submitted to the Plant.

\section{Plant material and treatment}

Seeds of G. hirsutum, Marie-Galante 85 (MG-85) race obtained from the Institute of Cotton Research, Chinese Academy of Agricultural Sciences (ICR-CAAS), were used. The seeds were first delinted using sulfuric acid then grown on moist absorbent paper for four days. The germinated seedlings were then transferred to a hydroponic set up with Hoagland nutrient solution (Hoagland \& Arnon, 1950) in the climate-controlled greenhouse with $16 \mathrm{~h} \mathrm{light} / 8 \mathrm{~h}$ dark and the temperature at $28^{\circ} \mathrm{C}$, day and $25^{\circ} \mathrm{C}$ night as previously described (Kirungu et al., 2019). At the three-leaf stage, the cotton seedlings were subjected to osmotic stress by adding to the Hoagland nutrient solution 17\% of glycol PEG-6000 and $300 \mathrm{mM}$ of sodium chloride for drought and salt treatment, respectively. To ensure the results were reliable, the untreated plants were used as the control. We collected samples in three biological replicates from the leaves, stem, and root tissues for RNA extraction at $0 \mathrm{~h}, 3 \mathrm{~h}, 6 \mathrm{~h}, 9 \mathrm{~h}, 12 \mathrm{~h}, 24 \mathrm{~h}$, and $48 \mathrm{~h}$ of post-stress exposure.

\section{RNA extraction and real-time RT-qPCR assays}

The RNAprep Pure Plant kit (Tiangen, Beijing, China) was used for RNA extraction by following its instructions. RNA's quality and concentration were determined using agarose gel electrophoresis and spectrophotometric analysis. The RNA with the correct concentration and purity was then converted to cDNA. The cDNA was prepared using EasyScript First-strand cDNA Synthesis SuperMix (TransGene, Beijing, China). The primers were designed using primer 5, list attached (Supplementary Table S1), and the cotton GhActin gene forward sequence 5'ATCCTCCGTCTTGACCTTG3' and reverse sequence 5'TGTCCGTCAGGCAACTCAT3' was used as the internal control. The real-time quantitative polymerase chain reaction (RT-qPCR) was performed as previously described by Lu et al. (Lu et al., 2019). The fold change was analyzed using the $2^{-\Delta \Delta C t}$ method (Livak \& Schmittgen, 2001).

\section{Validation of Gh_D01G0234 gene through Virus-Induced Gene Silencing (VIGS), under drought and salt stress conditions.}

Fragment of the coding DNA sequence of RPL14B ( $405 \mathrm{bp}$ ) was retrieved from the cotton functional genome database (https://cottonfgd.org/search/) and used to design its specific primer using the primer primer5 tool. The gene-specific primer, forward sequence: CGAGCTCCACGTGTTCCCAAGAAGAAGA, and Reverse sequence: CCTCGAG TTGCTTGATGACTCCAGACCT was amplified using $\mathrm{G}$. hirsutum CDNA by PCR. The product was then cloned into the EcoR1 and BamH1 sites of the Tobacco Rattle Virus vector (pTRV) to generate pTRV: RPL14B. The recombinant was then transformed into A. tumefaciens LBA4404 strain using freeze and thaw method (Dupadahalli, 2020). Preparation of the bacteria inoculum and inoculation to the plants' cotyledon was done as described by Corbin et al. (Corbin et al., 2017). For 
reliable results, we inoculated some plants with the silenced gene inoculum (pTRV: RPL14B), Phytoene desaturase (pTRV: PDS) to determine the effectiveness of the silencing, while other plants were inoculated with empty vector (pTRV: 00) and plants without any inoculum were denoted as the wild type and represented the control (Yang, Nyangasi, Odongo, Xu, \& Pu, 2019). Drought and salt stress simulation was done at the three-leaf stage by adding to the Hoagland solution $300 \mathrm{mM}$ sodium chloride for salt treatment and 17\% (w/v) of PEG-6000 for drought treatment (Yang et al., 2019). Sampling for physiological, morphological, and biochemical analysis was done on the leaf, stem, and root before treatment and $24 \mathrm{~h}$ after drought and salt treatment. The samples were then placed in liquid nitrogen. After that, they were kept at $-80^{\circ} \mathrm{C}$.

\section{Physiological and morphological analysis under drought and salt stress conditions}

Samples in three bio-replicates were collected before treatment and $24 \mathrm{~h}$ post-stress treatment. We assessed the susceptibility and tolerance of silenced and non-silenced plants to stress by determining the physiological and morphological parameters. Excised Leaf Water Loss (ELWL), relative leaf water content (RLWC), and cell membrane stability (CMS) were the physiological parameters determined as previously described (Cai et al., 2019). Briefly, to determine ELWL, fresh leaf samples were weighed (FW), then put on the bench for $24 \mathrm{~h}$ under normal room temperature then weighed to get the wilted weight (WW). Afterward, the leaves were put inside a $50^{\circ} \mathrm{C}$ dry oven for two days then weighed to get the leaf dry weight (DW). Using this formula, we calculated the ELWL. To determine RLWC, the leaf sample's fresh weight was measured (FW) and then put in distilled water under normal room temperature for $24 \mathrm{~h}$, surface dried, and weighed to get saturated weight (SW). After that, put inside a $50^{\circ} \mathrm{C}$ dry oven for two days and weighed to get the leaf dry weight (DW). Calculation of RLWC was done using the formula, . Cell membrane stability (CMS) was determined by quantifying plant electrolyte or ion leakage. Leaves from the silenced plants and control with uniform diameter and weighing $0.5 \mathrm{~g}$ were put in tubes containing $5 \mathrm{ml}$ distilled water and kept in the dark for $24 \mathrm{~h}$. Then we measured the electrical conductivity (L1). The leaves were then autoclaved at $70^{\circ} \mathrm{C}$ for 30 minutes and left to cool, and the electrical conductivity (L2) was measured. The formula used to calculate the cell membrane stability is; (L1 - L0)/(L2 - L0) $\times 100$ (L0 is the conductivity of distilled water).

The morphological parameters measured were the plant height $(\mathrm{PH})$, root length $(\mathrm{RL})$, shoot fresh weight (SFW), and root fresh weight (RFW). Plant height and root length were measured in centimeters, while shoot fresh weight and root fresh weight was measured in grams.

\section{Evaluation of Oxidants and Antioxidants enzymes in VIGS plants and wild types under drought and salt stress conditions.}

We further evaluated the effect of simulated osmotic stress on the silenced and control plants by quantifying the oxidant enzyme and antioxidant enzyme activities. We evaluated the antioxidants and oxidants enzyme activities on VIGS plants, plants transfused with empty vector and wild type under drought and salt stress conditions. Extraction and spectrometric analysis of the oxidants and 
antioxidants enzymes were carried out using their respective assay kits supplied by Beijing Solarbio Science and Technology, China, according to the manufacture's protocols.

\section{Results}

\section{Physio-chemical properties of the RPL14B protein in cotton}

In evaluating the physio-chemical properties of the RPL14B proteins encoded by the RPL14B genes in G. hirsutum, G. arboreum and G.raimondii cotton species, the proteins were found to exhibit varied features. However, one common feature to all the species is negative hydropathy gravy that ranged between -0.322 and -0.657 . They were indicating that all the proteins encoded by RPL14B were hydrophilic. The other properties varied among the cotton species (Table S1). For instance, molecular weights of RPL14B genes in $\mathrm{G}$. hirsutum ranged from $6.232 \mathrm{kDa}$ to $15.723 \mathrm{kDa}$, and isoelectric point (pl) value ranged from 10.86 to 11.55. While in G. raimondii and G. arboreum, the molecular weight ranged from $10.714 \mathrm{kDa}$ to 18.105 $\mathrm{kDa}$, the isoelectric point (pl) 11.5 to 17. The diploid cotton had a higher molecular weight and isoelectric point compared to the tetraploid cotton species ranged from-0.322 to -0.629 .

\section{Phylogenetic analysis and chromosomal distribution of RPL14B protein in cotton}

The cotton RPL14B proteins sequences were analyzed to determine the evolutionary pattern of RPL14B proteins. Using MEGA7, a phylogenetic tree was constructed from the RPL14B protein sequences aligned using ClustalX. The RPL14B were grouped into three groups (Figure 1). The RPL 14B genes are distributed in thirteen chromosomes in $G$. hirsutum. In the A genome is; $A 01, A 02, A 07, A 09, A 10, A 11, A 12$, while in the D genome, D01, D02, D03, D07, D09, D10, D11, and D13 with two genes mapped within the scaffold (Figure 2).

\section{RPL14B gene structure, domain and conserved motif}

The cotton $R P L 14 B$ genes had several introns in their gene structure (Figure $3 \mathrm{~A}-\mathrm{C}$ ). Few introns were associated with stress-responsive genes, as seen in previous studies on other stress-responsive genes in cotton, such as dehydrin (Kirungu et al., 2019). Thus, low intron interruption in RPL 14B genes indicated that they are involved in stress acclimation mechanisms in cotton. Ribosomal domain(s) is an essential component of all the RPs. They all have RPL14-KOW conserved domains at their N-terminal that enable them to interact with other proteins. RPL14B has several motifs (Figure 3D-F). The motifs contain invariant glycine residue, which is composed of alternating blocks of hydrophilic and hydrophobic residue.

\section{Cis-Acting regulatory element, subcellular localization prediction and $\mathrm{GO}$ analysis}

All the RPL 14B genes across the three cotton species have stress-related cis-regulatory elements. The cisregulatory elements obtained are shown in (Figure 4A); all the identified cis-regulatory elements are involved in phytohormones and abiotic stress response. RPL14B genes were predicted to be sub localized in the mitochondrion, nucleus, and endoplasmic reticulum. However, they are abundant in the nucleus, 
especially in the G. hirsutum (Figure 4B). GO enrichment analysis showed that this gene has all the GO functions: the molecular function, biological process and cellular component. Cellular components associated with this gene are ribosomes and intracellular organelles. RPL14B is part of metabolic and cellular cell processes. The molecular function is the structural molecule activity of the cell (Figure $4 \mathrm{C}$ ( $\mathrm{i}-$ iii)).

\section{Expression of RPL14B genes in upland cotton under drought and salt stress}

The expression analysis of GhRPL $14 B$ genes was assayed through RT-qPCR. The expression levels of the genes under drought and salt stress were differential (Figure 5). In the leaf, the expression was higher from $12 \mathrm{~h}$ to $48 \mathrm{~h}$ under both drought and salt stress. While in the roots, the expression was high from the onset of stress, at $3 \mathrm{~h}$, they were highly expressed, and the expression was differential onwards up to $48 \mathrm{~h}$ under stress conditions

\section{Evaluation of the efficiency of RPL14B gene silencing}

The effectiveness of silencing of the RPL14B gene in cotton was evaluated using the phytoene desaturase (PDS) gene. Previous research has shown that PDS can be used as a positive control to evaluate the effectiveness of silencing a particular gene. Plants infiltrated with PDS tend to exhibit a photobleached leaf phenotype that extends to the stem. In this experiment, the plants infused with pTRV2: PDS showed albino trait after 2 weeks of post-inoculation. The leaves and upper part of the stem were exhibiting this chlorotic/ bleached phenotype. RT-qPCR analysis to determine expression levels of $R P L 14 B$ gene in silenced plants and wild type showed that the gene expression was lower in silenced plants relative to wild type. This demonstrates that this gene's silencing was successful, and the vector used was effective (Figure 6).

\section{Evaluation of Morphological and physiological traits of the VIGS-plant and wild type (WT) under drought and salt conditions}

RPL14B silenced plants and the control plants under normal conditions exhibited no physiological or morphological changes. However, when the plants were subjected to drought and salt stress conditions, the plants showed some wilting elements and indicated that they were stressed (Figure 7(A-C)). The plant height $(P H)$, root length $(R L)$, and root fresh weight (RFW) exhibited a significant difference between the VIGS plants the controls (Figure 7(D-F). The silenced Gh_D01G0234 cotton leaves showed a significant reduction in RLWC relative to wild type and TRV2:00 leaves. Whereas there was a relative increase in ELWL and ion leakage compared to wild type and TRV2:00 leaves. An increase in ion leakage and ELWL demonstrates this gene's silencing compromises the plant's effectiveness in tolerating drought stress (Figure 7(G-I).

\section{Oxidant and Antioxidant Enzymes Assay}

The plants were further analyzed for the levels of oxidant and antioxidant enzymes. These enzymes were assayed on the VIGS plant's leaf tissue and wild type under drought and salt stress conditions. The 
antioxidants (POD and CAT) level in VIGS plants was significantly reduced compared to the wild type. In contrast, the oxidant (MDA) level was significantly high in VIGS plants compared to wild type (Figure 8). This result demonstrates that silencing of RPL14B compromises the plant's ability to tolerate drought and salt stress.

\section{Discussion}

Abiotic stress factors like drought and salt have exacerbated cotton production with an estimated loss of $70 \%$. Plants being sessile initiates signaling pathways, activation of transcriptional factors and eventually expression of stress-responsive genes, all this is to ensure plant survival. It is imperative to identify genes involved in sustaining plant growth and development during abiotic stress to improve their productivity further. The RPL gene family has been known to be involved with the housekeeping of the ribosome. However, recent studies have demonstrated that ribosomal protein has evolved (Horiguchi et al., 2012), and they are involved in extra ribosomal activities like biotic (Li, 2019) and abiotic stress tolerance (Mukhopadhyay, Reddy, Singla-Pareek, \& Sopory, 2011). Under abiotic stress, plants increase the production of protein as a metabolic response (Song et al., 2014). Under stress conditions, protein in the plant undergoes denaturation, and it is crucial to maintain the homeostasis between protein synthesis and degradation to ensure a normal metabolic process (Byrne, 2009). Several studies have been done in several plants, for instance, rice (Moin et al., 2017), tobacco (Liu et al., 2014), and Arabidopsis (Sormani, Masclaux-Daubresse, Daniele-Vedele, \& Chardon, 2011), whereby many RPL genes are upregulated in response to abiotic stress suggesting that they are involved in maintaining or improving protein biosynthesis enabling plants to acclimatize to stress. All these studies demonstrated that ribosomal protein is involved in abiotic stress tolerance.

In this study, the phylogenetic tree results showed that the RPL proteins have diverse distribution and could perhaps have a common origin. Similar results have been shown in various subtypes of the LEA proteins, in which the various classes showed wider distribution across the three cotton genomes, $A, D$ and (AD), (Magwanga, Lu, Kirungu, Dong, et al., 2018). Three cotton species had different numbers of genes, which had the RPL14B functional domain. 26, 5 and 8 genes were found in $G$. hirsutum (AD), $G$. raimondii (D) and $G$. arboreum (A), respectively. All the genes were found to have negative hydropathy (GRAVY); that is, they are hydrophilic. The GRAVY values are important protein property because it indicates the protein's behavior in relation to water. Several proteins that were hydrophilic have been found to participate in stress acclimation. For instance, research done on the LEA2 gene in cotton found that they were hydrophilic and conferred to enhance drought stress acclimation (Magwanga, Lu, Kirungu, Dong, et al., 2018).

The nucleus has an integral role in cell functioning. This involves the regulation of gene expression under different internal and external conditions and regulates the synthesis of proteins. The majority of $R P L 14 B$ genes were located in the nucleus. Their presence in the nucleus indicates that these gene activities are crucial in cell activities, especially abiotic stress. RPL14B has the RPL14-KOW motif at its N-terminal. KOW motif has been identified in some large ribosomal proteins (Kyrpides, Woese, \& Ouzounis, 1996). 
This motif contains invariant glycine residue, which is composed of alternating blocks of hydrophilic and hydrophobic residue. KOW motif is common among other families of ribosomal protein like RPL 19, 21.2, 24b, and 26; this shows the evolutionary relationship between this gene and KOW motif family. KOW motif is involved in protein-protein interaction and links Ribosomal protein with transcription factors that respond to abiotic stress (Moin et al., 2016).

This protein interacts with other genes like RPL 3, 4, 18, 19, 23 and ubiquitin. Some of these genes are involved in stress responses. For instance, overexpression of RPL23A in rice enhanced the water use efficiency of the plants under abiotic stress (Moin et al., 2017). Several studies have demonstrated that ubiquitin is an abiotic stress signaling molecule in plants, and they promote protein interaction in response to abiotic stress (Stone, 2014). RPL14 interacts with RPL3 and RPL19 together with the rRNA of the RPL and upholds the ribosome's stability (Tiller et al., 2012). Previous studies have shown that RPL19 under drought conditions was upregulated and enhanced tolerance to drought stress. It is also involved thymidylate synthase gene splicing and regulation of protein synthesis during photosynthesis (Semrad \& Schroeder, 1998). For plant adaptive responses to drought and salinity, transcription of stress-related genes associated with tolerance mechanisms and pathways is essential. Under drought and salt stresses, stress-related genes interact with other genes and induce their transcription to initiate appropriate responses. Therefore the interaction of these genes enhances stress response and tolerance.

The cis-regulatory elements upstream of the transcription factor region play an active role in activating and suppressing genes in response to stress conditions (Zhao, Xia, Liu, \& Ma, 2014). The presence of several stress-responsive cis-regulatory elements in the putative promoter regions of the RPL14B gene reveals that this gene activity alleviates the plant's stress effects. In addition to abiotic stresses, elements that respond to phytohormones were identified. ABRE (Abscisic acid-responsive element), TGACG-motif and CGTCA-motif responsive to MeJa, TCA- motif responsive to salicylic acid, TGA- motif responsive to Auxin were identified. Previous research on $R P L$ stress-responsive gene families in rice identified similar cis-regulatory (Moin et al., 2017; Saha et al., 2017). This suggests RPL14B gene activities enhance plant's adaptation and tolerance to abiotic stress and participate in signal pathways during abiotic stress conditions.

Virus-induced gene silencing is a versatile tool for functional characterization and has been extensively utilized to study gene function in different plants (Corbin et al., 2017). We utilized this tool to determine whether silencing RPL14B in cotton interferes with the plant's stress acclimation mechanisms. The silenced Gh_D01G0234 plants exhibited a susceptibility phenotype compared to the control plants. The fresh leaf weight, shoot fresh weight, fresh root weight, RLWC, and chlorophyll content of VIGS plants were lower than that of control plants (empty vector and wild plants), while ELWL and ion leakage were higher in VIGS plants compared to control plants. Similar observations were observed in which plants exhibited wilting behaviors when exposed to either osmotic or salinity stress conditions (Fathi \& Tari, 2016). This result indicates that VIGS plants experienced reduced water retention and photosynthetic activities. Thus, they were more susceptible to drought and salt stress compared to control plants. The transpiration rate in plants under stress increases when its stress tolerance mechanisms are

Page $11 / 25$ 
compromised (Suzuki, Rivero, Shulaev, Blumwald, \& Mittler, 2014). Biochemical analysis showed a higher concentration level of MDA and a lower level of POD, and CAT, in VIGS plants relative to control plants. A higher amount of oxidant means VIGS plants were experiencing oxidative stress under drought and salt stress conditions. Drought stress results in the upregulation of oxidants; this is due to the lack of homeostasis between oxidants and antioxidants. Oxidative stress results in the production of reactive oxygen species (ROS). The ROS are incredibly toxic and can cause damage to the plant tissues and eventually cell death. Plants use ROS to aid in the signal transduction process in response to various stimuli and offer the plant defense to abiotic stress (Mehla, Sindhi, Josula, Bisht, \& Wani, 2017). Oxidants and antioxidants have been used as biochemical markers for drought stress in various studies; upregulation of oxidants and downregulation of antioxidants indicates the plant in under stress.

\section{Conclusions}

This study provides an insight into the role of the $R P / 14 B$ gene during drought and salt stresses conditions. The RP genes are involved in stabilizing the ribosomes and interacting with other genes, enhancing plant acclimation to unfavorable conditions. The presence of cisregulatory elements and increased expression of the RPL14B gene during drought and salt stress proves that $R P L$ genes have evolved and are involved in extra ribosomal activities. Therefore, more studies on the $R P L$ genes should be done to understand these genes' mechanisms in response to cotton stress conditions.

\section{Declarations}

\section{Acknowledgements}

We are grateful for the support and guidance accorded by Prof Liu Fang throughout the research work.

\section{Authors' contributions}

Margaret L. Shiraku: Methodology, Investigation, Software, Writing - review \& Editing: Richard Odongo Magwanga: Conceptualization, Writing - review \& Editing: Xiaoyan CAl: Methodology, Resources, Validation. Joy Nyangasi Kirungu: Investigation, Software. Yanchao Xu: Investigation, Software. Teame Gereziher Mehari ${ }^{1}$ : Investigation, Software. Yuqing Hou: Resources, Investigation. Yuhong Wang: Project administration, Resources, Investigation. Renhai Peng: Supervision, review. Kunbo Wang: Validation, Funding acquisition, Supervision. Zhongli Zhou: Conceptualization, Funding acquisition, Supervision, Writing - review \& editing. Fang Liu Conceptualization, Validation, Funding acquisition, Supervision, Writing - review \& editing.

\section{Funding}

The National Natural Science Foundation of China (31621005, 31530053, and 31671745), the Agricultural Science and Technology Innovation Program of the Chinese Academy of Agricultural Sciences financially sponsored this research program 


\section{Ethics approval and consent to participate}

No ethical nor consent to participate in this research was sought.

\section{Consent for publication}

No consent to publish the work was sort.

\section{Competing interests}

The authors declare no any form of competing interest.

\section{References}

Bailey, T. L., Boden, M., Buske, F. A., Frith, M., Grant, C. E., Clementi, L., ... Noble, W. S. (2009). MEME Suite: Tools for motif discovery and searching. Nucleic Acids Research. https://doi.org/10.1093/nar/gkp335

Byrne, M. E. (2009). A role for the ribosome in development. Trends in Plant Science, 14(9), 512-519. https://doi.org/10.1016/j.tplants.2009.06.009

Cai, X., Magwanga, R. O., Xu, Y., Zhou, Z., Wang, X., Hou, Y., ... Wang, K. (2019). Comparative transcriptome, physiological and biochemical analyses reveal response mechanism mediated by CBF4 and ICE2 in enhancing cold stress tolerance in Gossypium thurberi. AoB PLANTS, 11(6), 1-17. https://doi.org/10.1093/aobpla/plz045

Campbell, B. T., Saha, S., Percy, R., Frelichowski, J., Jenkins, J. N., Park, W., ... Podolnaya, L. (2010). Status of the global cotton germplasm resources. Crop Science. https://doi.org/10.2135/cropsci2009.09.0551

Chaillou, T. (2020). Translational Control of Muscle Mass Ribosome specialization and its potential role in the control of protein translation and skeletal muscle size, 599-607.

https://doi.org/10.1152/japplphysiol.00946.2018

Chen, C., Chen, H., Zhang, Y., Thomas, H. R., Frank, M. H., He, Y., \& Xia, R. (2020). TBtools: An Integrative Toolkit Developed for Interactive Analyses of Big Biological Data. Molecular Plant. https://doi.org/10.1016/j.molp.2020.06.009

Corbin, C., Lafontaine, F., Sepúlveda, L. J., Carqueijeiro, I., Courtois, M., Lanoue, A., ... Courdavault, V. (2017). Virus-induced gene silencing in Rauwolfia species. Protoplasma, 254(4), 1813-1818. https://doi.org/10.1007/s00709-017-1079-y

Des Roches, S., Post, D. M., Turley, N. E., Bailey, J. K., Hendry, A. P., Kinnison, M. T., ... Palkovacs, E. P. (2018). The ecological importance of intraspecific variation. Nature Ecology and Evolution. https://doi.org/10.1038/s41559-017-0402-5 
Dupadahalli, K. (2020). A modified freeze - thaw method for efficient transformation of Agrobacterium tumefaciens, (September 2007).

Fathi, A., \& Tari, D. B. (2016). Effect of Drought Stress and its Mechanism in Plants. International Journal of Life Sciences, 10(1), 1-6. https://doi.org/10.3126/ijls.v10i1.14509

Haigler, C. H., Betancur, L., Stiff, M. R., \& Tuttle, J. R. (2012). Cotton fiber: a powerful single-cell model for cell wall and cellulose research. Frontiers in Plant Science. https://doi.org/10.3389/fpls.2012.00104

Hoagland, D. R., \& Arnon, D. I. (1950). Preparing the nutrient solution. The Water-Culture Method for Growing Plants without Soil, 347, 29-31. https://doi.org/citeulike-article-id:9455435

Horiguchi, G., Van Lijsebettens, M., Candela, H., Micol, J. L., \& Tsukaya, H. (2012). Ribosomes and translation in plant developmental control. Plant Science, 191-192, 24-34.

https://doi.org/10.1016/j.plantsci.2012.04.008

Hortona, P., Park, K. J., Obayashi, T., \& Nakai, K. (2006). Protein subcellular localization prediction with WoLF PSORT. In Series on Advances in Bioinformatics and Computational Biology.

https://doi.org/10.1142/9781860947292_0007

Hu, B., Jin, J., Guo, A. Y., Zhang, H., Luo, J., \& Gao, G. (2015). GSDS 2.0: An upgraded gene feature visualization server. Bioinformatics. https://doi.org/10.1093/bioinformatics/btu817

Kim, C.-K., Oh, J.-H., Na, J.-K., Cho, C., Kim, K.-H., Yu, G. E., \& Kim, D.-Y. (2019). The Genes Associated with Drought Tolerance by Multi-Layer Approach in Potato. Plant Breeding and Biotechnology, 7(4), 405-414. https://doi.org/10.9787/pbb.2019.7.4.405

Kim, K., Park, S., Chung, Y., Chung, C., Kim, J., \& Lee, J. (2004). Molecular cloning of low-temperatureinducible ribosomal proteins from soybean, 55(399), 1153-1155. https://doi.org/10.1093/jxb/erh125

Kirungu, J. N., Magwanga, R. O., Pu, L., Cai, X., Xu, Y., Hou, Y., ... Liu, F. (2019). Knockdown of Gh_A05G1554 (GhDHN_03) and Gh_D05G1729 (GhDHN_04) Dehydrin genes, Reveals their potential role in enhancing osmotic and salt tolerance in cotton. Genomics, (September).

https://doi.org/10.1016/j.ygeno.2019.11.003

Kumar, S., Stecher, G., \& Tamura, K. (2016). MEGA7: Molecular evolutionary genetics analysis version 7.0. molecular biology and evolution. Molecular Biology and Evolution.

Kyrpides, N. C., Woese, C. R., \& Ouzounis, C. A. (1996). KOW: A novel motif linking a bacterial transcription factor with ribosomal proteins. Trends in Biochemical Sciences. https://doi.org/10.1016/S09680004(96)30036-4

Li, S. (2019). Regulation of Ribosomal Proteins on Viral Infection. Cells, 8(5), 508. https://doi.org/10.3390/cells8050508 
Liu, X.-D., Xie, L., Wei, Y., Zhou, X., Jia, B., Liu, J., \& Zhang, S. (2014). Abiotic Stress Resistance, a Novel Moonlighting Function of Ribosomal Protein RPL44 in the Halophilic Fungus Aspergillus glaucus. Applied and Environmental Microbiology. https://doi.org/10.1128/aem.00292-14

Livak, K. J., \& Schmittgen, T. D. (2001). Analysis of relative gene expression data using real-time quantitative PCR and the 2- $\Delta \Delta C$ T method. Methods. https://doi.org/10.1006/meth.2001.1262

Lu, P., Magwanga, R. O., Kirungu, J. N., Hu, Y., Dong, Q., Cai, X., ... Liu, F. (2019). Overexpression of Cotton a DTX/MATE Gene Enhances Drought, Salt, and Cold Stress Tolerance in Transgenic Arabidopsis. Frontiers in Plant Science, 10(March). https://doi.org/10.3389/fpls.2019.00299

MAGWANGA, R. O., LU, P., KIRUNGU, J. N., CAI, X., ZHOU, Z., AGONG, S. G., ... LIU, F. (2020). Identification of QTLs and candidate genes for physiological traits associated with drought tolerance in cotton. Journal of Cotton Research, 3(1), 1-33. https://doi.org/10.1186/s42397-020-0043-0

Magwanga, R. O., Lu, P., Kirungu, J. N., Diouf, L., Dong, Q., Hu, Y., ... Liu, F. (2018). GBS mapping and analysis of genes conserved between gossypium tomentosum and gossypium hirsutum cotton cultivars that respond to drought stress at the seedling stage of the BC2F2generation. International Journal of Molecular Sciences, 19(6). https://doi.org/10.3390/ijms19061614

Magwanga, R. O., Lu, P., Kirungu, J. N., Dong, Q., Hu, Y., Zhou, Z., ... Liu, F. (2018). Cotton Late Embryogenesis Abundant ( LEA2) Genes Promote Root Growth and Confer Drought Stress Tolerance in Transgenic Arabidopsis thaliana . G3\&amp;\#58; Genes/Genomes/Genetics. https://doi.org/10.1534/g3.118.200423

Mehla, N., Sindhi, V., Josula, D., Bisht, P., \& Wani, S. H. (2017). An introduction to antioxidants and their roles in plant stress tolerance. In Reactive Oxygen Species and Antioxidant Systems in Plants: Role and Regulation under Abiotic Stress. https://doi.org/10.1007/978-981-10-5254-5_1

Moin, M., Bakshi, A., Madhav, M. S., \& Kirti, P. B. (2017). Expression profiling of ribosomal protein gene family in dehydration stress responses and characterization of transgenic rice plants overexpressing RPL23A for water-use efficiency and tolerance to drought and salt stresses. Frontiers in Chemistry, 5(NOV), 1-16. https://doi.org/10.3389/fchem.2017.00097

Moin, M., Bakshi, A., Saha, A., Dutta, M., Madhav, S. M., \& Kirti, P. B. (2016). Rice Ribosomal Protein Large Subunit Genes and Their Spatio-temporal and Stress Regulation. Frontiers in Plant Science, 7(August), 120. https://doi.org/10.3389/fpls.2016.01284

Mukhopadhyay, P., Reddy, M. K., Singla-Pareek, S. L., \& Sopory, S. K. (2011). Transcriptional downregulation of rice rpL32 gene under abiotic stress is associated with removal of transcription factors within the promoter region. PLOS ONE. https://doi.org/10.1371/journal.pone.0028058 
Oluoch, G., Zheng, J., Wang, X., Khan, M. K. R., Zhou, Z., Cai, X., ... Wang, K. (2016). QTL mapping for salt tolerance at seedling stage in the interspecific cross of Gossypium tomentosum with Gossypium hirsutum. Euphytica. https://doi.org/10.1007/s10681-016-1674-6

Rogalski, M., Schöttler, M. A., Thiele, W., Schulze, W. X., \& Bock, R. (2008). Rpl33, a nonessential plastidencoded ribosomal protein in tobacco, is required under cold stress conditions. Plant Cell. https://doi.org/10.1105/tpc.108.060392

Rogers, G. M., Poore, M. H., \& Paschal, J. C. (2002). Feeding cotton products to cattle. Veterinary Clinics of North America - Food Animal Practice. https://doi.org/10.1016/S0749-0720(02)00020-8

Saha, A., Das, S., Moin, M., Dutta, M., Bakshi, A., Madhav, M. S., \& Kirti, P. B. (2017). Genome-wide identification and comprehensive expression profiling of ribosomal protein small subunit (RPS) genes and their comparative analysis with the large subunit (RPL) genes in rice. Frontiers in Plant Science, 8(September), 1-21. https://doi.org/10.3389/fpls.2017.01553

Semrad, K., \& Schroeder, R. (1998). A ribosomal function is necessary for efficient splicing of the T4 phage thymidylate synthase intron in vivo. Genes and Development, 12(9), 1327-1337.

https://doi.org/10.1101/gad.12.9.1327

Singh, V., Kendall, R. J., Hake, K., \& Ramkumar, S. (2013). Crude oil sorption by raw cotton. Industrial and Engineering Chemistry Research. https://doi.org/10.1021/ie4005942

Song, J., Wei, X., Shao, G., Sheng, Z., Chen, D., Liu, C., ... Hu, P. (2014). The rice nuclear gene WLP1 encoding a chloroplast ribosome L13 protein is needed for chloroplast development in rice grown under low temperature conditions. Plant Molecular Biology, 84(3), 301-314. https://doi.org/10.1007/s11103013-0134-0

Sormani, R., Masclaux-Daubresse, C., Daniele-Vedele, F., \& Chardon, F. (2011). Transcriptional regulation of ribosome components are determined by stress according to cellular compartments in Arabidopsis thaliana. PLOS ONE, 6(12). https://doi.org/10.1371/journal.pone.0028070

Stone, S. L. (2014). The role of ubiquitin and the $26 \mathrm{~S}$ proteasome in plant abiotic stress signaling. Frontiers in Plant Science. https://doi.org/10.3389/fpls.2014.00135

Suzuki, N., Rivero, R. M., Shulaev, V., Blumwald, E., \& Mittler, R. (2014). Abiotic and biotic stress combinations. New Phytologist, 203(1), 32-43. https://doi.org/10.1111/nph.12797

Thompson, J. D., Gibson, T. J., \& Higgins, D. G. (2003). Multiple Sequence Alignment Using ClustalW and ClustalX. Current Protocols in Bioinformatics. https://doi.org/10.1002/0471250953.bi0203s00

Tian, T., Liu, Y., Yan, H., You, Q., Yi, X., Du, Z., ... Su, Z. (2017). AgriGo v2.0: A GO analysis toolkit for the agricultural community, 2017 update. Nucleic Acids Research. https://doi.org/10.1093/nar/gkx382 
Tiller, N., Weingartner, M., Thiele, W., Maximova, E., Schöttler, M. A., \& Bock, R. (2012). The plastid-specific ribosomal proteins of Arabidopsis thaliana can be divided into non-essential proteins and genuine ribosomal proteins. Plant Journal. https://doi.org/10.1111/j.1365-313X.2011.04791.x

Wang, L., He, S., Dia, S., Sun, G., Liu, X., Wang, X., ... Du, X. (2021). Industrial Crops \& Products Alien genomic introgressions enhanced fiber strength in upland cotton (Gossypium hirsutum $L$.). Industrial Crops \& Products, 159(November 2020), 113028. https://doi.org/10.1016/j.indcrop.2020.113028

Yang, X., Nyangasi, J., Odongo, R., Xu, Y., \& Pu, L. (2019). Knockdown of GhIQD31 and GhIQD32 increases drought and salt stress sensitivity in Gossypium hirsutum Plant Physiology and Biochemistry Knockdown of GhIQD31 and GhIQD32 increases drought and salt stress sensitivity in Gossypium hirsutum, (September). https://doi.org/10.1016/j.plaphy.2019.09.027

Zhao, T., Xia, H., Liu, J., \& Ma, F. (2014). The gene family of dehydration responsive element-binding transcription factors in grape (Vitis vinifera): Genome-wide identification and analysis, expression profiles, and involvement in abiotic stress resistance. Molecular Biology Reports.

https://doi.org/10.1007/s11033-013-3004-6

Zou, C., Sun, K., Mackaluso, J. D., Seddon, A. E., Jin, R., Thomashow, M. F., \& Shiu, S. H. (2011). Cisregulatory code of stress-responsive transcription in Arabidopsis thaliana. Proceedings of the National Academy of Sciences of the United States of America. https://doi.org/10.1073/pnas.1103202108

\section{Figures}




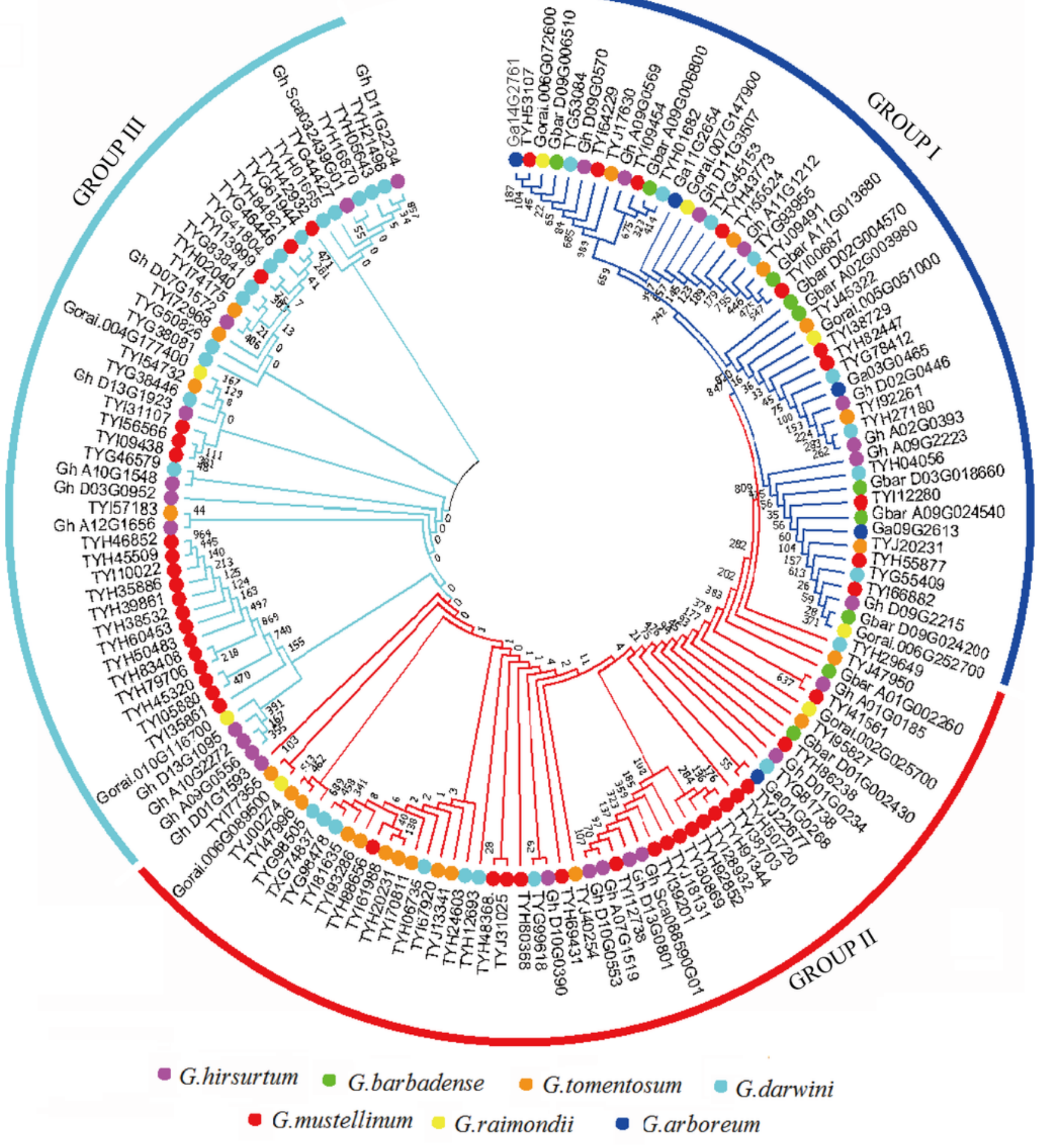

\section{Figure 1}

Phylogenetic tree analysis of RPL14 proteins in G. hirsutum, G. raimondii, G. arboreum, G. tomentosum, G. mustelinum, G. barbadense, and G. darwinii 
A

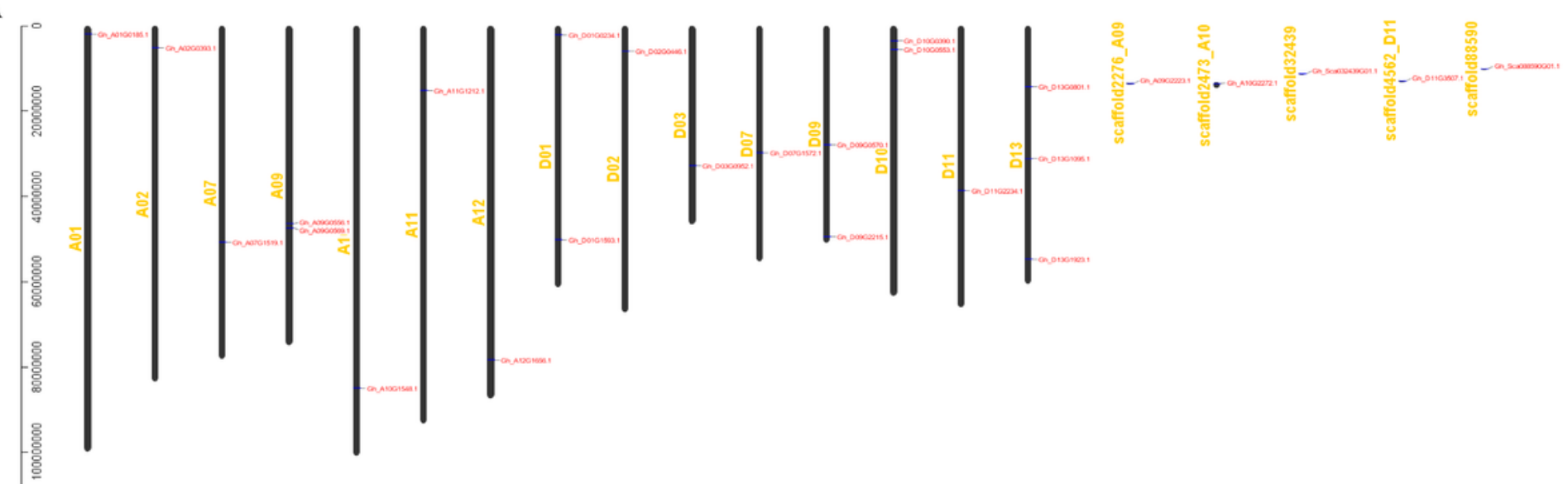

B

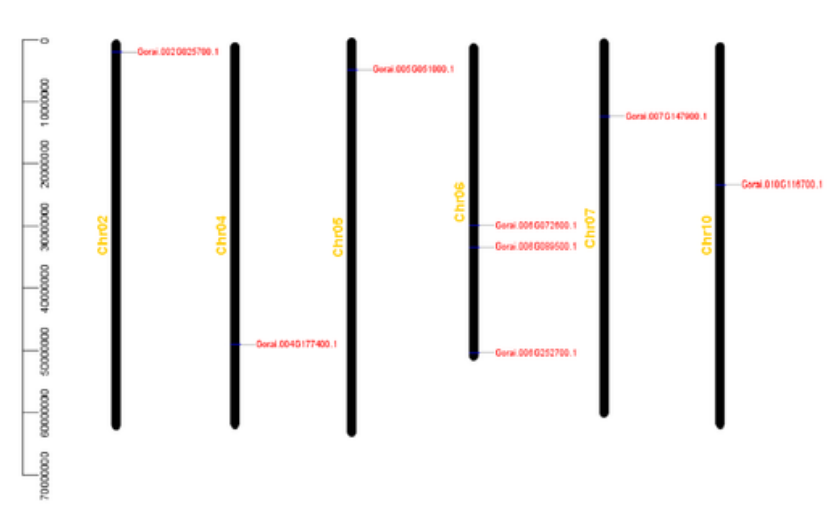

C

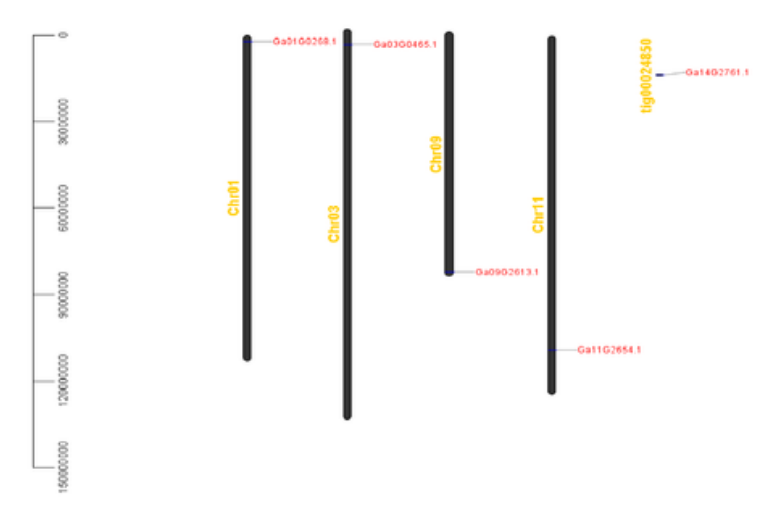

Figure 2

The distribution of RPL14B genes in chromosomes. (A) G. hirsutum (B) G.raimondii and (C) G. arboreum. 
$1 \mathrm{~A}$

catarcossu

Ge. 10200393.1

Ga. 10091519.1

Ga.ancoss.

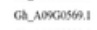

Gatarcins:

GaAloGises.

Ga-Alocenz:

Ga,aiscisose

G. Doncoesu.

Ga porgissy.

Ga. poscosest.

G. Doncisn?:

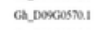

Ga.bosc2sis:

Ca. Drocoses
Ga. Dhocess

Ga. Dhocossiu

capucserot.

Ge. Discosol.1

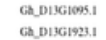

Ga.plyGise.1

Ga. scasssocent

Legend:

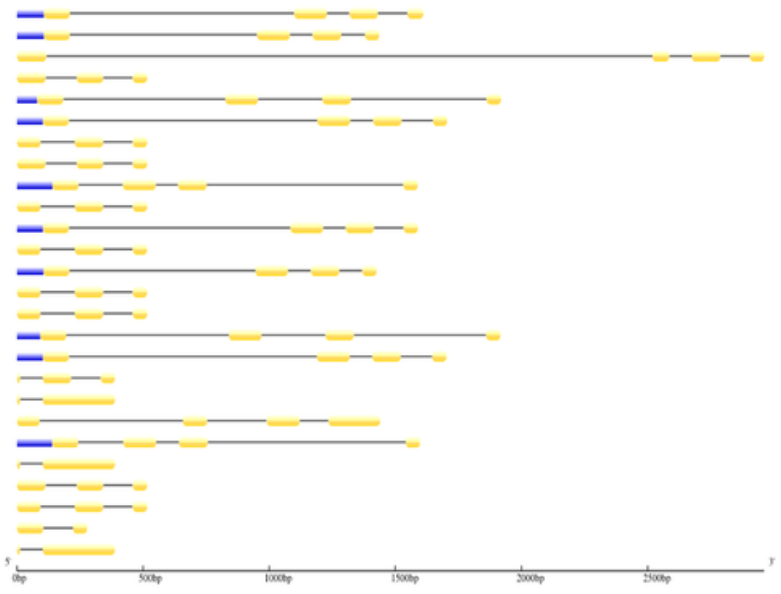

2A

Motir 3

Gh_A0200393.1 늘

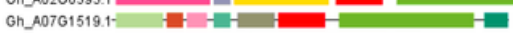

Gh_A0960556.1- 들

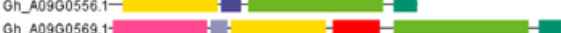

Gh_A0960569.1 -

Gh_A0962223.1 A1061548.1-

Gh_A10G1548.1-

Gh_A10G2272.1- 들

Gh_A1161212.1

Gh_A1201656.1-

on_D0100234.1 글

Oh_D0101593.1- 들

Oh_D0200446.1

Gh_D03G0952.1-

Gh_D0701572.1-

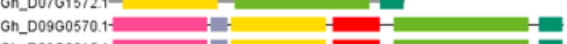

On_D0902215.1

Gh_D10G0390.1-

Oh_D1000553.1. 늘

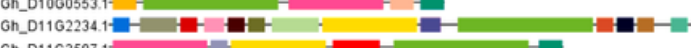

Gh_D1103507.1 들

Gh_D1360801.1-

Oh_D1361095.1-

On_Sca032439601.1-

Oh_Sca088590601.1늘

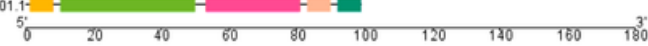

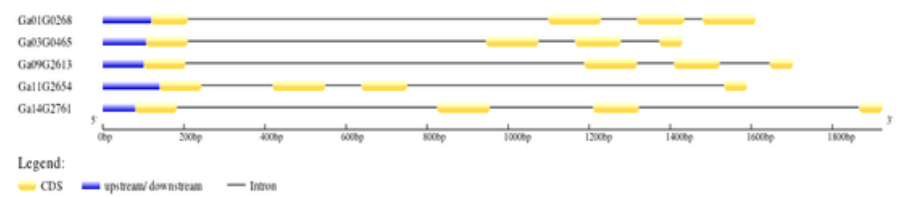

C

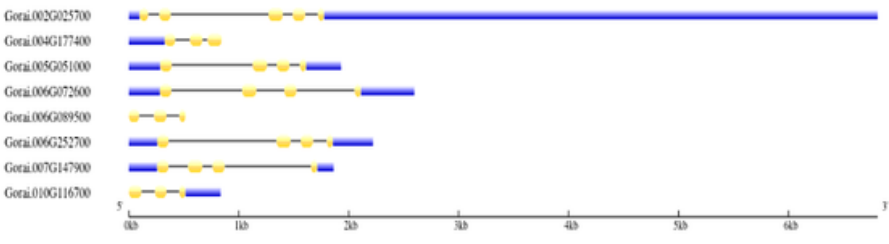
Legend:

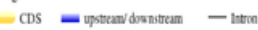

B

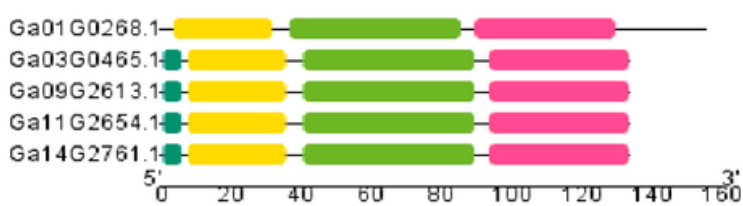

Motif 2

Matif 1

Matif 3

Matif 4

$\mathrm{C}$

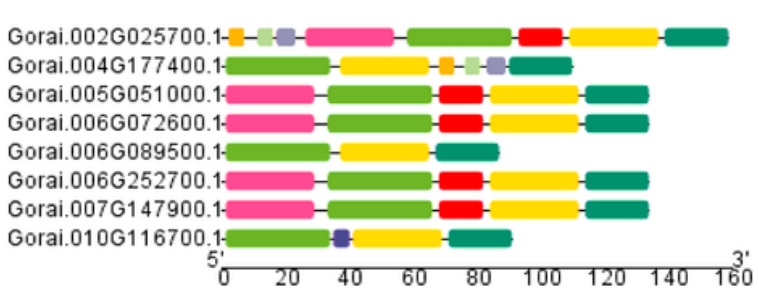

Motif 8

Motif 7

Motif 6

Motif 3

Motif 1

Motif 5

Motif 2

Motif 4

Motif 9

\section{Figure 3}

Gene structure and motif analysis of cotton RPL14B proteins. (A) Gene structure of genes in G. hirsutum, (B) gene structure of G.arboreum genes, (C) gene structure of G. raimondii genes, (D) motif present in G.hirsurtum, (E) motif present in G.arboreum and (F) motif present in G.raimondii. 

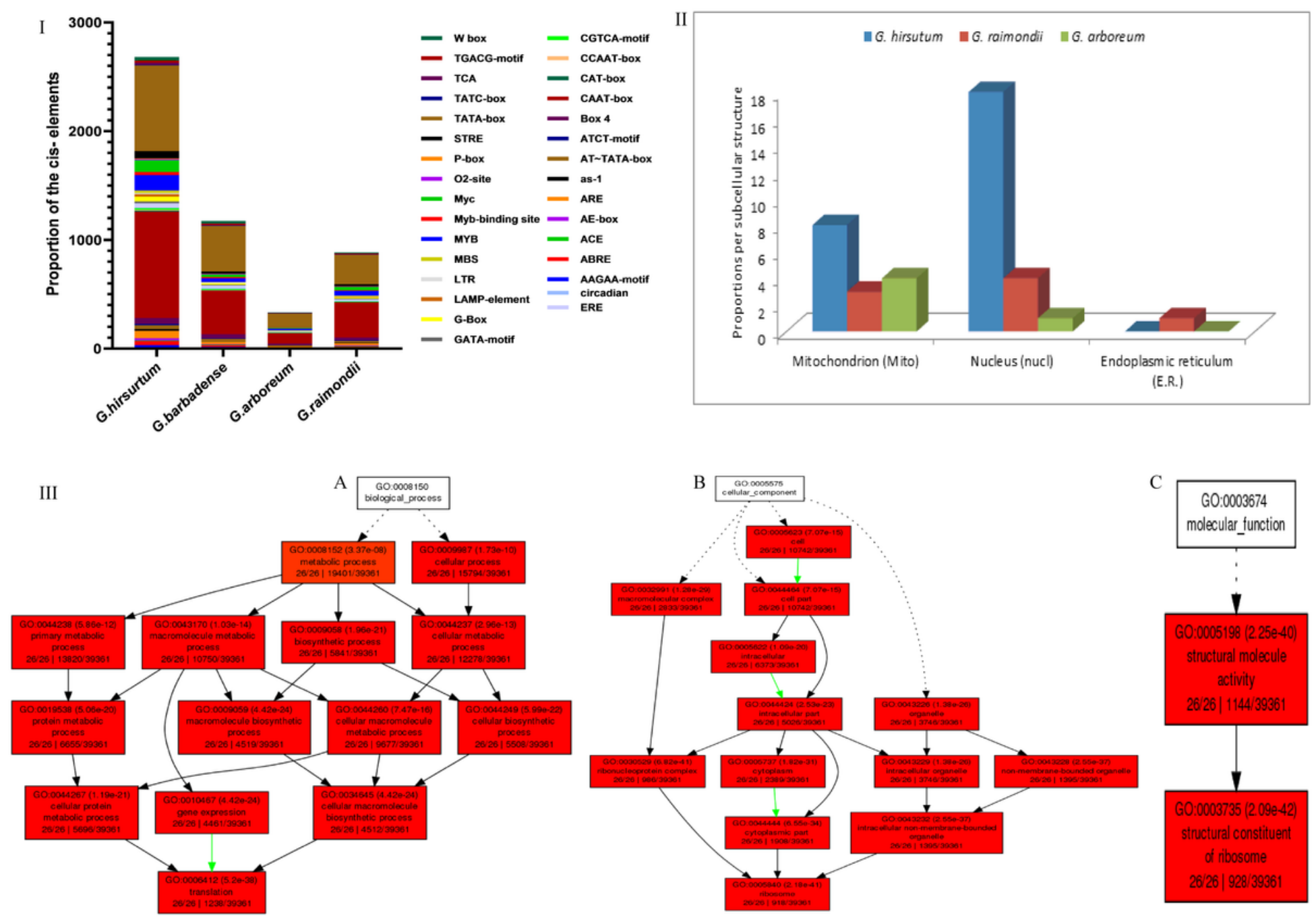

Figure 4

Cis-regulatory element, subcellular localization and GO analysis. (A). Cis-regulatory elements obtained from G. hirsutum, G. raimondii, G. arboreum, and G. barbadense. (B). 
A

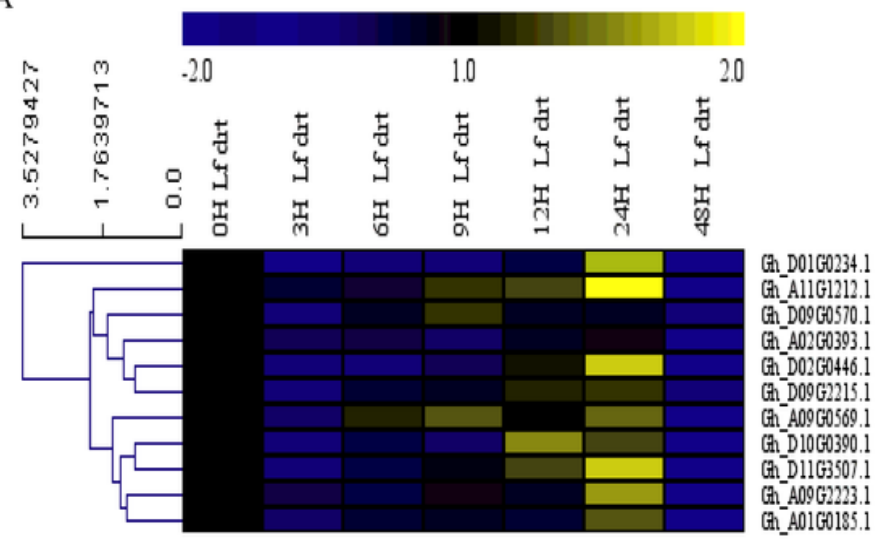

B

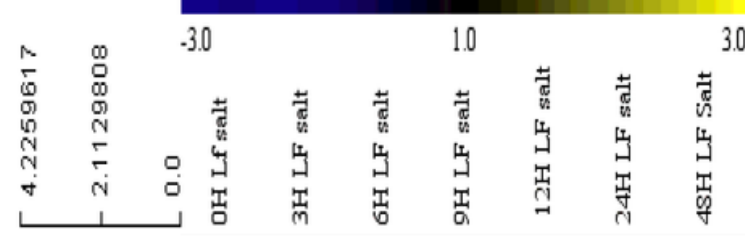

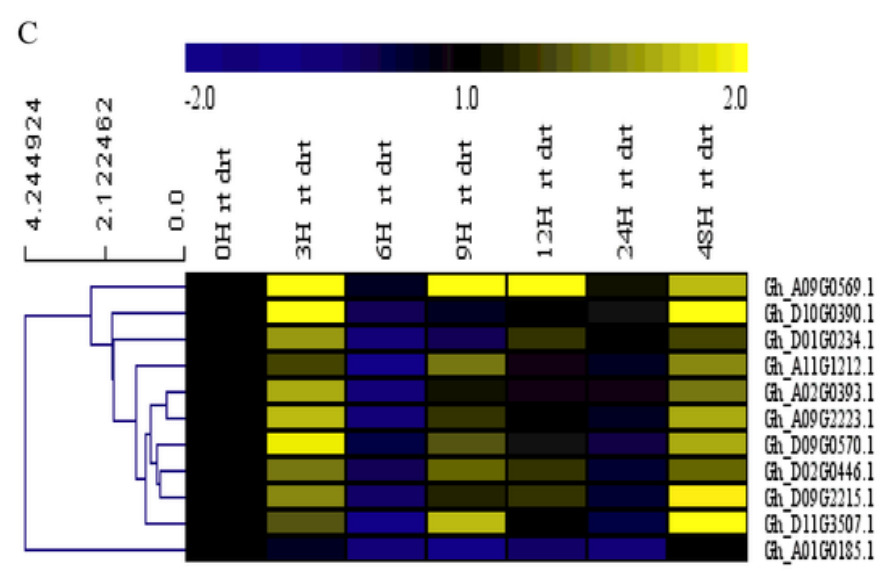

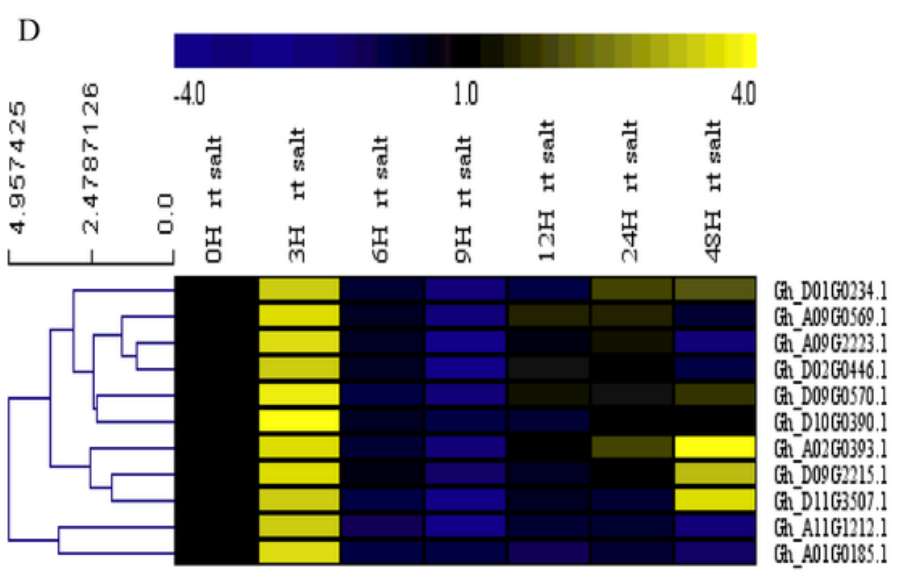

Figure 5

Differential expression of GhRPL14B genes under drought and salt stress. (A). Heat map of the RPL14 gene expression in the leaf under drought stress conditions. (B) Heat map of the RPL14 gene expression in the leaf under salt stress condition. (C) Heat map of the RPL14B gene expression in the root under drought stress conditions. (D) Heat map of the RPL14B gene expression in the root under salt stress condition. Yellow depicts a high expression of the genes, and blue depicts a low expression of genes. Black depicts no expression of the genes at a particular time. 
A

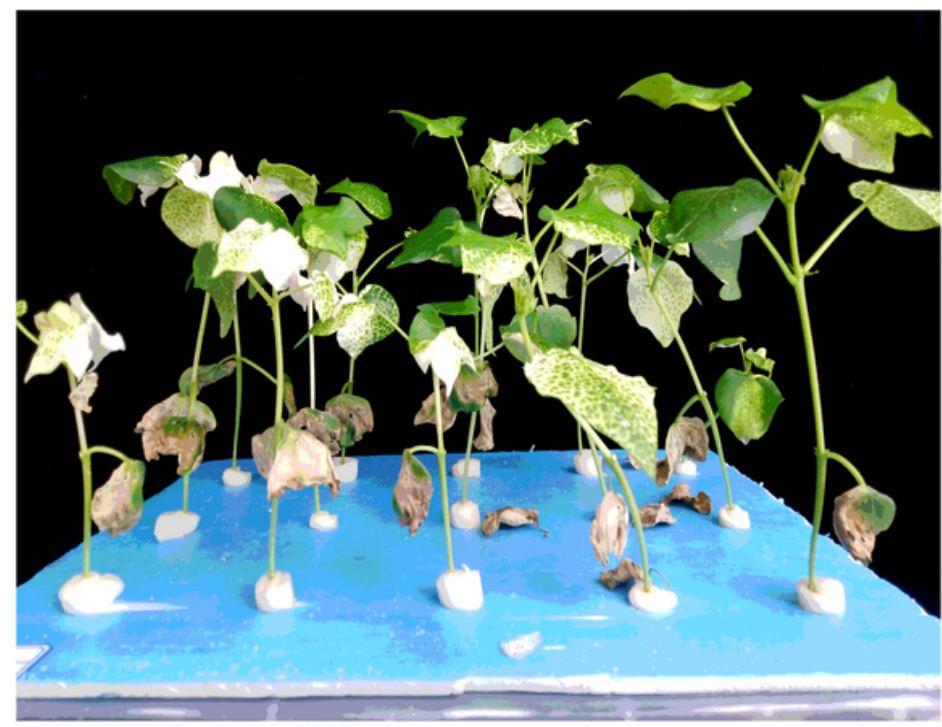

B

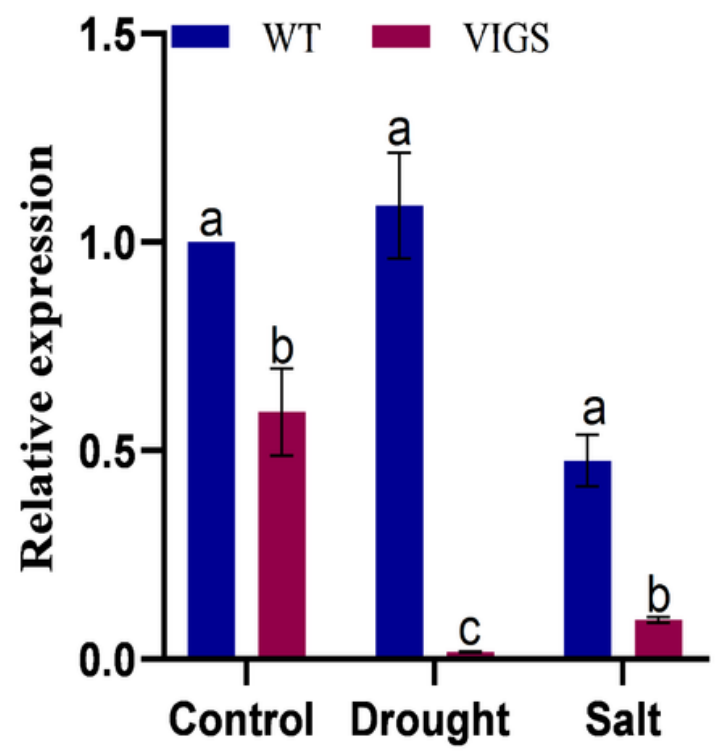

Figure 6

The efficiency of virus-induced gene silencing in the cotton seedlings. (A). The albino change on the plants under being inoculated with TRV: PDS after 14 days. (B). expression levels of the knocked gene in WT and VIGS-plants under normal conditions (control), drought and salt stress. 


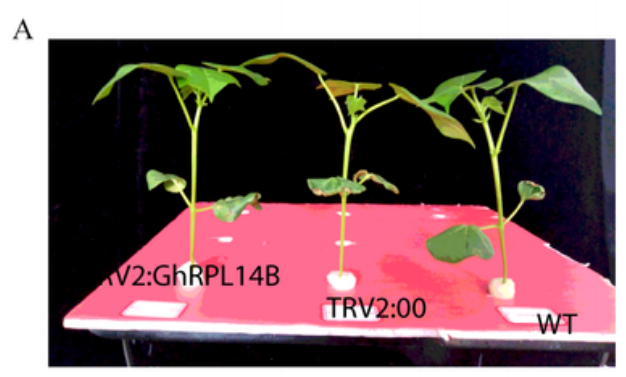

B

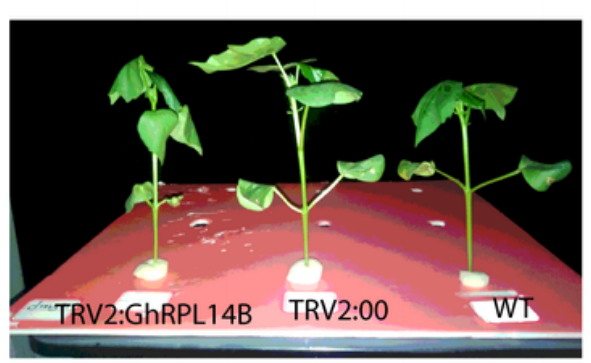

$\mathrm{E}$

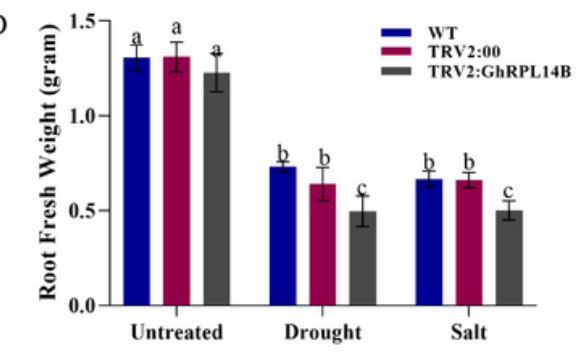

G

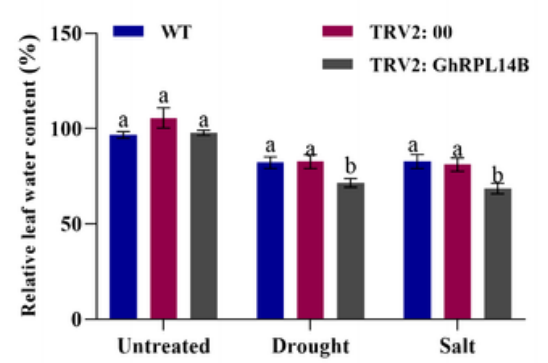

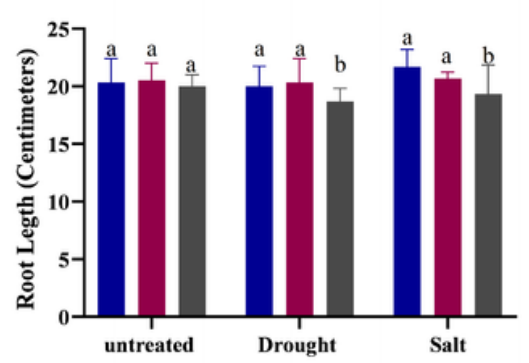

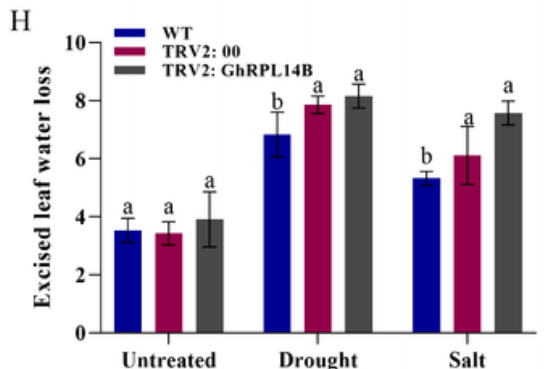

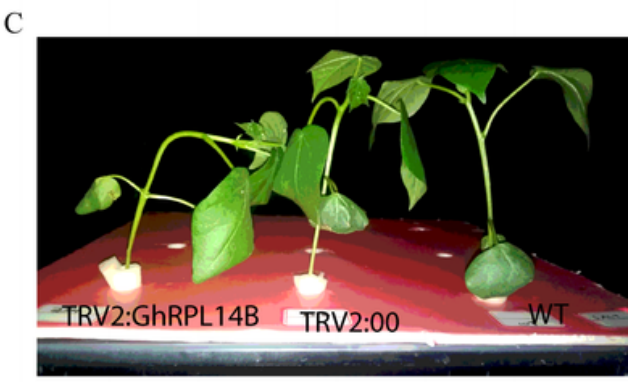
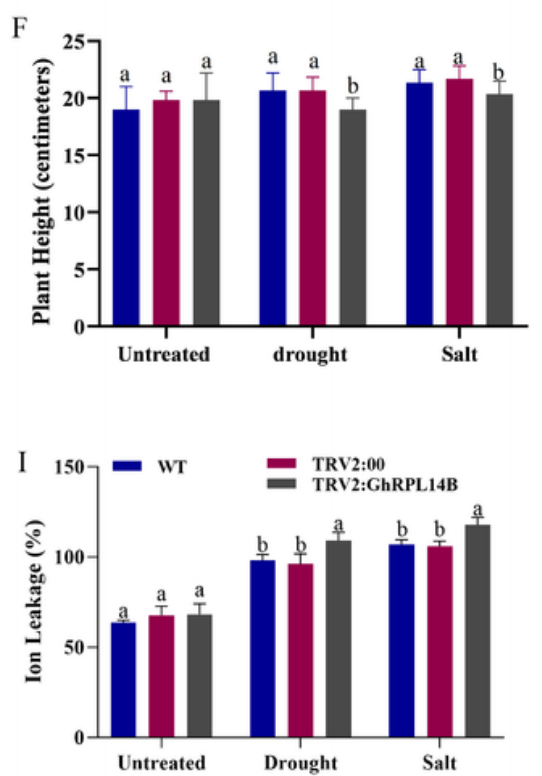

Figure 7

Morphological and physiological evaluation of the VIGS-plants and the wild types (WT) under drought and salt stress conditions. (A) Phenotype observation of TRV2: GhRPL14B, TRV2:00 and WT (Wild type) before stress treatment. (B) Phenotype observation of TRV2: GhRPL14B, TRV2:00 24h after 17\% PEG treatment. (C) Phenotype observation of TRV2: GhRPL14B, TRV2:00 and WT 24h after salt treatment. Morphological parameters, (D) Root fresh weight (RFW), (E) root length (RL), and (F) Plant height (PH). (G) Quantitative determination of relative leaf water content (RLWC), $(H)$ excised leaf water loss (ELWL), and (I) Quantitative determination of ion leakage as a measure of cell membrane stability (CMS). Bar indicates the standard error (SE). Different letters indicate the significant differences between wild type (WT) and VIGS-plants (ANOVA; $p<0.05)$. 

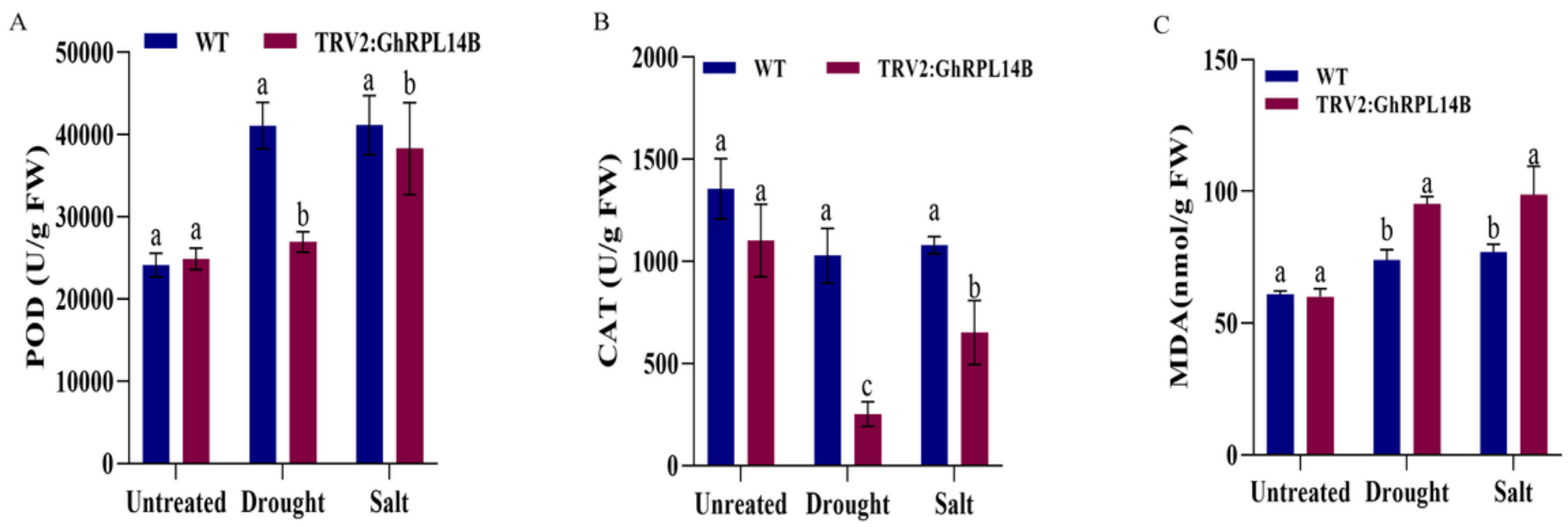

\section{Figure 8}

Biochemical assays of the oxidant and antioxidant in VIGS plants under drought and salt stress conditions. (A) Quantitative determination of peroxidase (POD), (B) Quantitative determination of catalase (CAT), and (C) quantitative determination of Malondialdehyde (MDA). The bar indicates a standard error (SE). Different letters indicate the significant differences between wild type and VIGSplants (ANOVA; $p<0.05)$.

\section{Supplementary Files}

This is a list of supplementary files associated with this preprint. Click to download.

- TableS1.xlsx

- Tables2.xls 